\title{
Fuzzy Local Binary Patterns for Ultrasound Texture Characterization
}

\author{
Dimitris K. Iakovidis ${ }^{1}$, Eystratios G. Keramidas ${ }^{2}$, \\ and Dimitris Maroulis ${ }^{3}$ \\ Dept. of Informatics and Telecommunications, University of Athens, \\ Panepistimioupolis, 15784, Athens, Greece \\ dimitris.iakovidis@ieee.org ${ }^{1}, \underline{\text { e.keramidas@di.uoa.gr }}{ }^{2}$, dmaroulis@di.uoa.gr $^{3}$
}

\begin{abstract}
B-scan ultrasound provides a non-invasive low-cost imaging solution to primary care diagnostics. The inherent speckle noise in the images produced by this technique introduces uncertainty in the representation of their textural characteristics. To cope with the uncertainty, we propose a novel fuzzy feature extraction method to encode local texture. The proposed method extends the Local Binary Pattern (LBP) approach by incorporating fuzzy logic in the representation of local patterns of texture in ultrasound images. Fuzzification allows a Fuzzy Local Binary Pattern (FLBP) to contribute to more than a single bin in the distribution of the LBP values used as a feature vector. The proposed FLBP approach was experimentally evaluated for supervised classification of nodular and normal samples from thyroid ultrasound images. The results validate its effectiveness over LBP and other common feature extraction methods.
\end{abstract}

Keywords: Fuzzy, Local Binary Patterns, Ultrasound, Thyroid nodules, Support Vector Machines.

\section{Introduction}

Ultrasonography is a very appealing modality for imaging both soft tissue and bony structures. Among the advantages of ultrasonography are low cost, real time imaging, no side effects, no invasion, and ease of use. Despite the advantages of diagnostic ultrasound, there are also two fundamental limitations, trade-off between resolution and attenuation, and the presence of speckle noise and artifacts. When dealing with superficial organs, like the thyroid gland, the resolution-attenuation limitation does not apply [1]. However, noise parameter affects strongly diagnosis based on a visual assessment of the structure and echogenicity, rather than objective measurement of certain quantitative properties.

There have been attempts towards less subjective techniques for the evaluation of ultrasound images, initially based on first-order statistical texture features. Local grey level histograms have been utilized in some of the first studies [2][3] to measure local textural information in ultrasound images. Several, more recent approaches [4-7], 
proposed features extracted from co-occurrence matrices, to characterize tissue on ultrasound images. Textural information encoded by means of Local Binary Patterns (LBP) [8], has also been applied to detect uniform texture patterns on ultrasound images [9-12]. However, the performance of feature extraction approaches such as cooccurrence matrices and Local Binary Patterns is adversely affected by noise and artifacts appearing in ultrasound images.

The aim of this study is to present and investigate the performance of a novel approach for texture characterization of ultrasound images. Such images exhibit some degree of uncertainty mainly due to speckle noise. However, most methodologies employed for encoding textural information have little tolerance to uncertainty. A texture descriptor, more capable of dealing with such textural information can be developed by incorporating fuzzy logic in the Local Binary Pattern methodology. This novel approach, called Fuzzy LBP (FLBP) enables more robust representation of texture than the crisp (original) LBP in the inherently noisy US images.

This paper is organized in four sections. In Section 2, the original LBP and the proposed FLBP feature extraction methods are described. In Section 3, a comparative experimental evaluation reveals the advantageous performance of the proposed method in comparison to other methods applied on real US images. In the last section a short discussion on results, conclusions and future perspectives are presented.

\section{Fuzzifying the Local Binary Patterns}

The LBP feature extraction method is a theoretically and computationally simple, and efficient methodology for texture analysis. The LBP operator was first introduced by Ojala et al. in 1996 [8], as a non-parametric, grey-scale invariant texture analysis model, which summarizes the local spatial structure of an image. This LBP operator was based on a $3 \times 3$ local neighborhood (Fig. 1a) representing the local texture around a central pixel. The value of each peripheral pixel of this neighborhood is thresholded by the value of the central pixel in two possible values $\{0,1\}$. A total of $2^{8}=256$ possible LBP codes can describe spatial binary patterns of $3 \times 3$ pixel neighborhoods.

In the LBP texture representation, a pattern is represented by a set of nine elements $P=\left\{p_{\text {center },} p_{0}, p_{1}, \ldots, p_{7}\right\}$, where $p_{\text {center }}$ represents the intensity value of the central pixel and $p_{\mathrm{i}}(0 \leq i \leq 7)$ represent the intensity values of the peripheral pixels (Fig. 1a). A $3 \times 3$ neighborhood can be characterized by a set of binary values $d_{i}(0 \leq i \leq 7)$ (Fig. $1 b)$, where

\begin{tabular}{|l|l|l|}
\hline$p_{0}$ & $p_{1}$ & $p_{2}$ \\
\hline$p_{3}$ & $p_{\text {center }}$ & $p_{4}$ \\
\hline$p_{5}$ & $p_{6}$ & $p_{7}$ \\
\hline
\end{tabular}

(a)

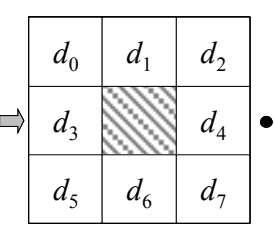

(b)

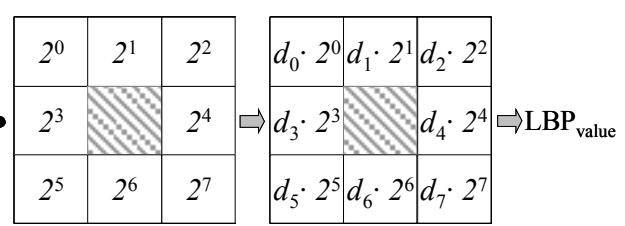

(c) (d)

Fig. 1. Local Binary Pattern computation scheme. 


$$
d_{i}= \begin{cases}1 & \text { if } p_{i} \geq p_{\text {center }} \\ 0 & \text { if } p_{i}<p_{\text {center }}\end{cases}
$$

Based on these binary values, for each neighborhood a unique LBP code can be derived as follows:

$$
L B P=\sum_{i=0}^{7} d_{i} \cdot 2^{i}
$$

Thus, the local microtexture information around a pixel, represented by a binary pattern, can be encoded by a single integer code $L B P \in[0,255]$.

Every pixel in an image generates a single LBP code. Then a histogram is created to collect up the occurrences of different LBP codes from all pixels in the image. This histogram forms the LBP feature vector, which characterize the image texture.

The LBP is based on hard thresholding of peripheral pixels, which makes texture representation sensitive to noise. In order to enhance the LBP approach so as to cope with the uncertainty introduced by the speckle noise, we have considered fuzzy logic, as a means to cope with inexactness and improve discrimination power of LBP approach in noise degraded images. Fuzzy logic resembles human decision making, with ability to finding precise solutions in approximate datasets.

The fuzzification of the LBP approach includes the transformation of the input variables to respective fuzzy variables, according to a set of fuzzy rules. To that direction, we introduce two fuzzy rules to describe the relation between the intensity values of the peripheral pixels $p_{i}$ and the central pixel $p_{\text {center }}$ of a $3 \times 3$ neighborhood as follows:

Rule $\boldsymbol{R}_{\boldsymbol{0}}$ : The smaller $p_{i}$ is, with respect to $p_{\text {center, }}$, the greater the certainty that $d_{i}$ is 0 .

Rule $\boldsymbol{R}_{\boldsymbol{I}}$ : The bigger $p_{i}$ is, with respect to $p_{\text {center }}$, the greater the certainty that $d_{i}$ is 1 .

According to the rules $R_{0}$ and $R_{1}$, two membership functions, $m_{0}()$ and $m_{1}()$, can be determined. Let function $m_{0}()$ define the degree to which $p_{i}$ has a smaller grey value than $p_{\text {center }}$, and thus define the degree to which $d_{i}$ is 0 . As a membership function $m_{0}()$ we consider the decreasing function (Fig. 2) defined as follows:

$$
m_{0}(i)= \begin{cases}0 & \text { if } p_{i} \geq p_{\text {center }}+T \\ \frac{T-p_{i}+p_{\text {center }}}{2 \cdot T} & \text { if } p_{\text {center }}-T<p_{i}<p_{\text {center }}+T \\ 1 & \text { if } p_{i} \leq p_{\text {center }}-T\end{cases}
$$




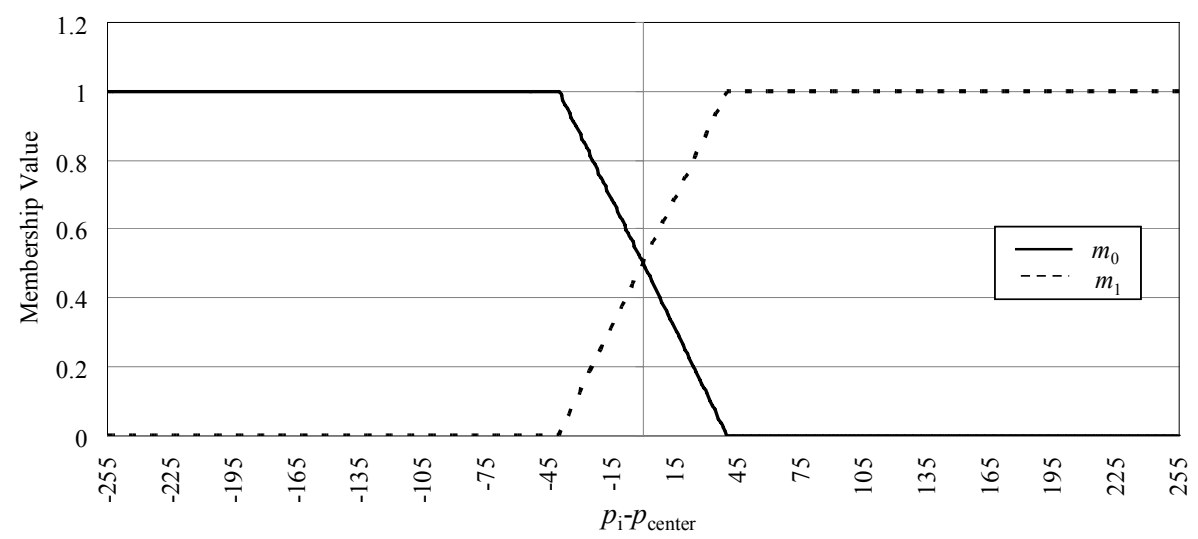

Fig. 2. Membership functions $m_{0}()$ and $m_{1}()$ for $T=40$, as a function of $p_{i}-p_{\text {center }}$.

On the other hand, membership function $m_{1}()$ defines the degree to which $p_{i}$ has a greater grey value than $p_{\text {center }}$, and thus define the degree to which $d_{i}$ is 1 . The membership function $m_{1}()$ considered is (Fig. 2):

$$
m_{1}(i)=1-m_{0}(i)
$$

For both $m_{0}()$ and $m_{1}(), T \in[0,255]$ represents a parameter that controls the degree of fuzziness.

Although for the original LBP operator a single LBP code characterizes a $3 \times 3$ neighbourhood, in the proposed FLBP approach, a neighbourhood can be characterized by more than one LBP code. Figure 3 presents an example of the FLBP approach, where two LBP codes characterize a $3 \times 3$ neighbourhood. The degree to which each LBP code characterizes a neighbourhood, depends on the membership functions $m_{0}()$ and $m_{1}()$. For a $3 \times 3$ neighbourhood, the contribution $C_{L B P}$ of each LBP code in a single bin of the FLBP histogram is defined as:

$$
C_{L B P}=\prod_{i=0}^{8} m_{d i}(i)
$$

where $d_{i} \in\{0,1\}$ and the LBP code can be obtained from Eq. 2. For each peripheral pixel, $d_{i}$ can be either 0 or 1 , with a grade of $m_{0}()$ or $m_{1}()$ respectively, forming different LBP codes with different contributions (Eq. 5). Thus, each $3 \times 3$ neighbourhood contributes to more than one bin of the FLBP histogram. The total contribution of a $3 \times 3$ neighbourhood to the bins of an FLBP histogram is:

$$
\sum_{L B P=0}^{255} C_{L B P}=1
$$




\begin{tabular}{|c|c|c|}
\hline 90 & 200 & 110 \\
\hline 180 & 142 & 150 \\
\hline 182 & 181 & 100 \\
\hline
\end{tabular}

(a)

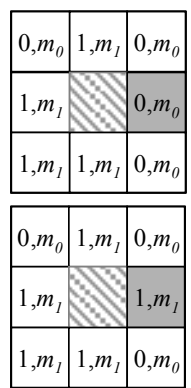

(b)

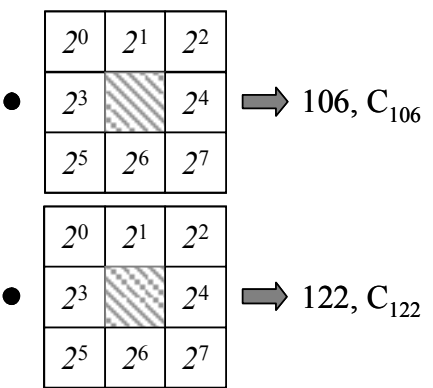

(c)

Fig. 3. A simple example of the FLBP computation schema on a $3 \times 3$ neighbourhood for $T=10$. (a) $3 \times 3$ neighbourhood. (b) Fuzzy thresholded values along with membership values. (c) Binomial weights. (d) LBP codes and corresponding contribution values.

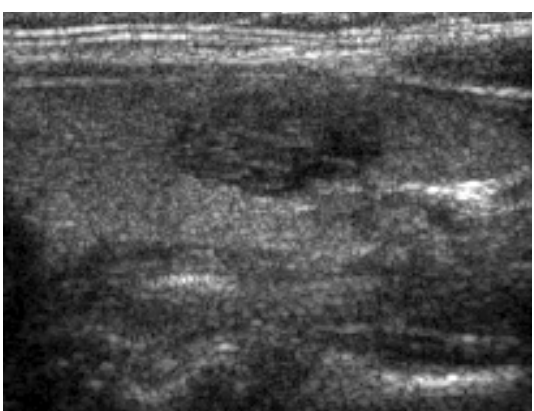

(a)

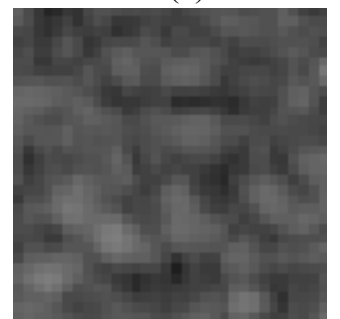

(c)

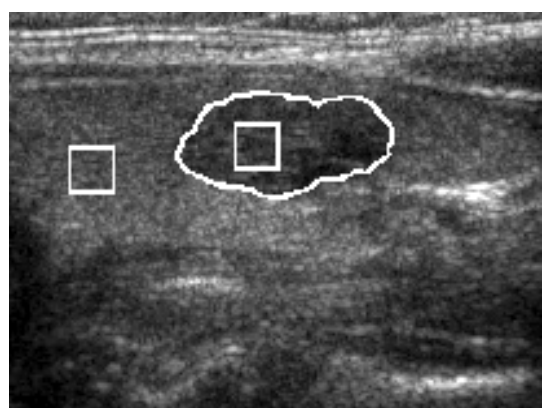

(b)

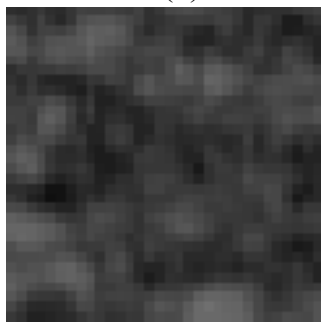

(d)

Fig. 4. (a) Example ultrasound image displaying a visible thyroid nodule. (b) The same image with the boundaries of the nodule marked and two square sampled blocks, acquired from normal parenchyma and from inside the nodule. (c) Square block sampled from normal parenchyma. (d) Square block sampled from nodular tissue.

Figures 5(a) and 5(c) illustrate two LBP histograms calculated from blocks sampled from image regions corresponding to normal (Fig. 4c) and nodular tissue (Fig. 4d), respectively. It can be observed that in these histograms 118 and 127 out of 255 bins have zero value respectively. This results in a small set of significant peaks that can be identified for each histogram. The corresponding FLBP histograms are 


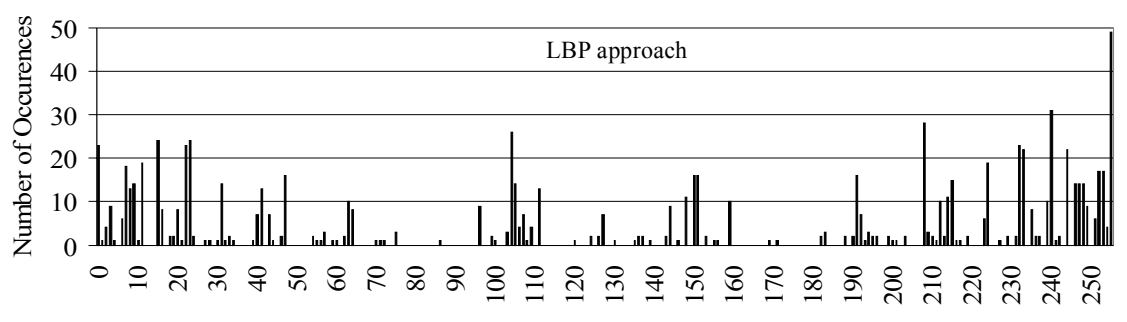

LBP Codes

(a)

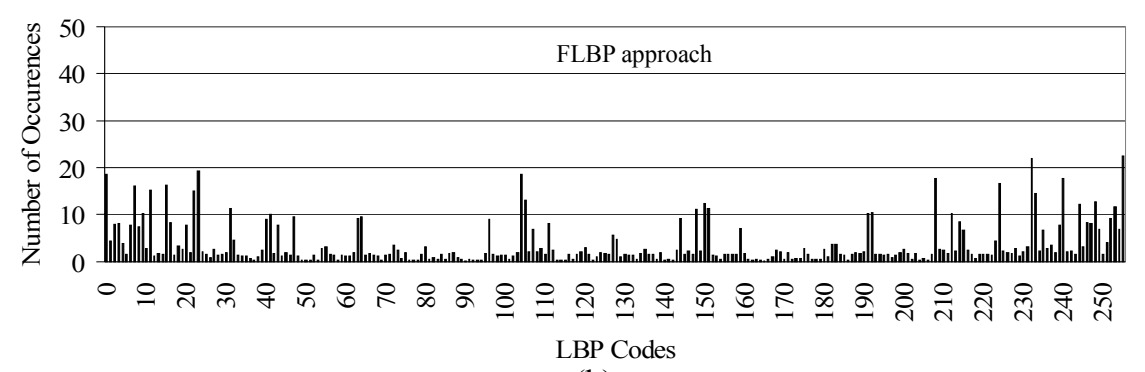

(b)

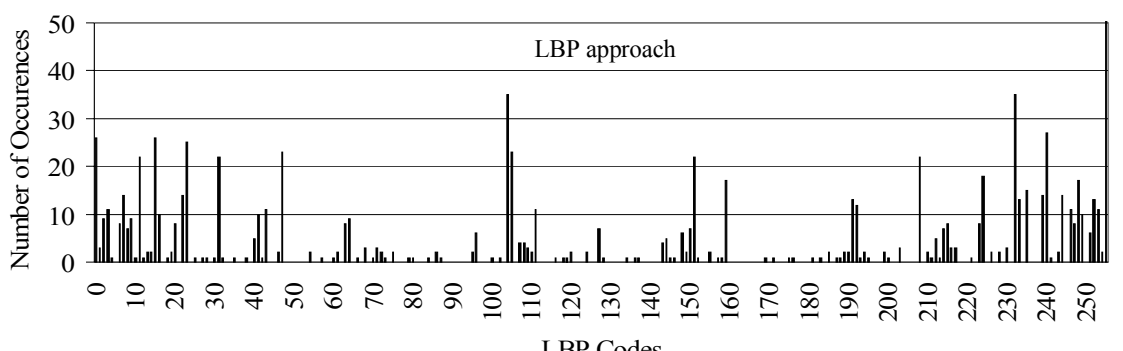

(c)

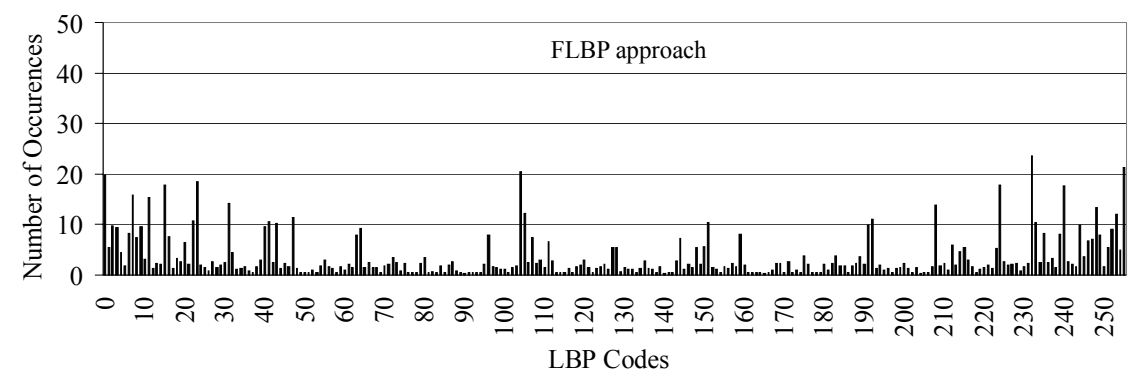

(d)

Fig. 5. Histograms obtained by LBP and FLBP approaches applied on images presented on Fig.4(c) and Fig.4(d). (a) LBP histogram from normal thyroid tissue. (b) FLBP histogram from normal thyroid tissue for $T=5$. (c) LBP histogram from nodular thyroid tissue. (d) FLBP histogram from nodular thyroid tissue for $T=5$. 
illustrated in Figs. 5(b) and 5(d). These histograms do not have bins with zero values and there are more spikes, though limited in magnitude. This indicates that FLBP histograms are more informative than LBP histograms. Considering that Shannon entropy is defined as

$$
H=-\sum_{L P B=0}^{255} p_{L B P} \cdot \log \left(p_{L B P}\right)
$$

where $p_{L B P}$ is the probability of the LBP-th pattern, the more diversified the signal, the higher the entropy, and the more the actual information. If all the bins have equal probability, the maximum entropy will be reached. Apparently, the FLBP histograms give greater of equal entropy than the crisp LBP histograms.

\section{Results}

For the evaluation of the feature extraction method proposed in this study a set of classification experiments were carried out on real thyroid ultrasound images. Ultrasound examinations were performed on 36 patients using a Philips HDI 5000 sonographic imaging system with an amplitude resolution of 8 bits (256 grey levels) and a spatial resolution of $470 \times 470$ pixels. The parameters of the sonograph were kept the same during all the examinations. A total of 65 thyroid ultrasound images with one or more nodules was acquired. From each image, blocks of $32 \times 32$ pixels were selected from inside the nodules and from the normal thyroid parenchyma (Fig. 4), forming a balanced set of 240 image samples.

The classification of the acquired samples was performed by a Support Vector Machine (SVM) [13], a classifier based on the principle of structural risk minimization that works well for high-dimensional input spaces and exhibit excellent generalization performance. On that ground we used four SVM classifiers utilizing linear, polynomial of second degree, radial basis, and sigmoid kernels respectively. The classification accuracy was estimated by 10 -fold cross validation [14].

Twenty FLBP feature sets were extracted by using different values for the fuzzification parameter $T$ in the range between zero and 20. For $T=0$, the crisp LBP values were obtained. As a baseline method to compare the classification results obtained by the proposed method we have considered the Co-occurrence Matrix (CM) approach used in [15]. The best results for the different kernel functions are illustrated in Fig. 6.

The maximum accuracy obtained is $84 \%$ and it was achieved with FLBP features for $T=5$ and polynomial kernel. The best results obtained with the LBP and with the $\mathrm{CM}$ features reached $62 \%$ and $70 \%$ respectively. Figure 6 shows that the FLBP approach performs better than the LBP and the CM approach regardless of the kernel used. 


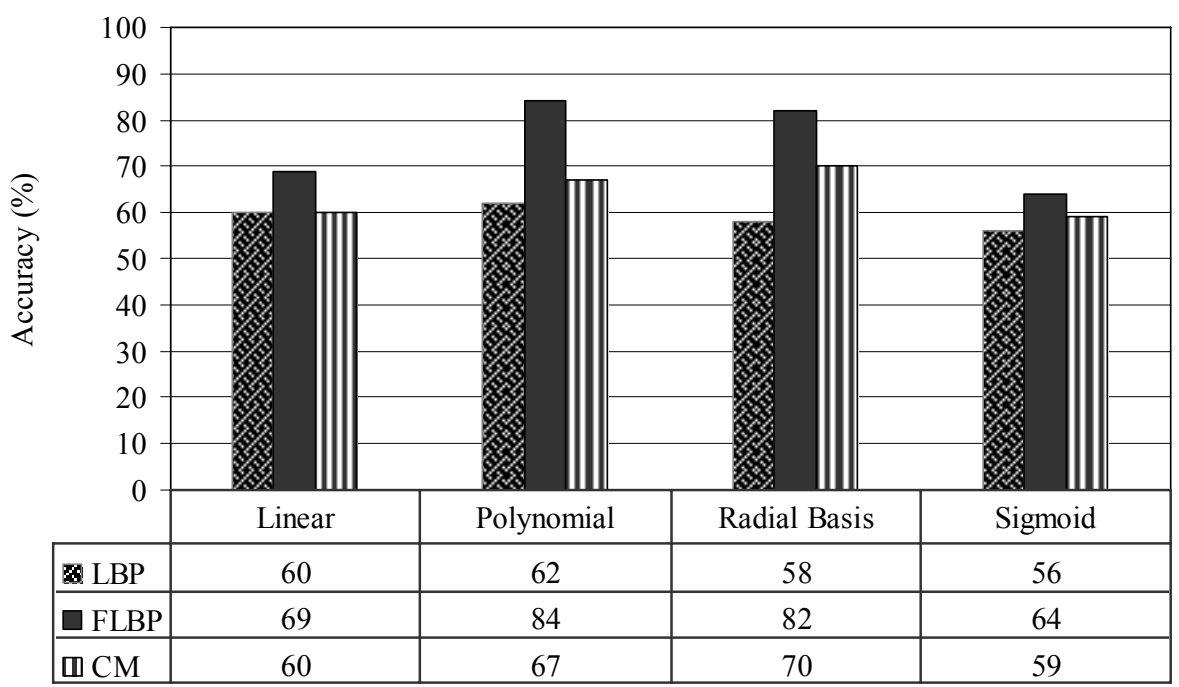

Fig. 6. Best classification results obtained with LBP, FLBP and co-occurrence matrix (CM) features using various SVM kernel functions.

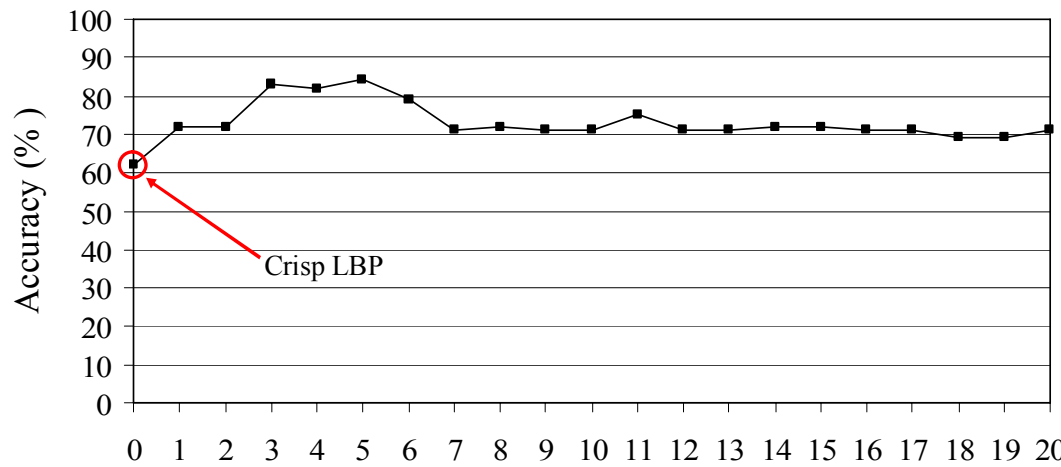

Fuzzification Parameter $T$

Fig. 7. Classification accuracy obtained with FLBP features for different values of the fuzzification parameter $T$ and polynomial SVM kernel.

Moreover, it should be noted that the FLBP approach performs better than the LBP approach for every $T>0$. The classification accuracies obtained for $T \geq 0$ with the polynomial kernel are illustrated in Fig. 7.

\section{Conclusions}

In this study a novel Fuzzy Local Binary Pattern (FLBP) operator has been proposed, for better representation of textures in ultrasound images in which uncertainty is introduced by inherent noise. The proposed approach was experimentally evaluated and compared with the crisp LBP and with the co-occurrence matrix approaches on a real dataset of nodular and normal thyroid tissue ultrasound images. The experimental classification results of this study demonstrate that the proposed FLBP approach: 
- can considerably improve texture representation in noisy US images.

- results in significantly higher classification performance, as compared to the CM approach.

Future work and perspectives include:

- Investigation of the performance of FLBP approach on ultrasound images acquired from different sonographs.

- Integration of the proposed approach to a system for the assessment of the thyroid gland.

\section{Acknowledgments}

We would like to thank Dr. N. Dimitropoulos, and EUROMEDICA S.A., Greece for the provision of the medical images. We would also like to thank Dr. G. Legakis for his continuous support and advice. This work was supported by the Greek General Secretariat of Research and Technology (25\%), the European Social Fund (75\%), and private sector, under the framework of Measure 8.3 of E.P. Antagonistikotita $-3^{\text {rd }}$ European Support Framework - PENED 2003 (grant no. 03-ED-662).

\section{References}

1. Bushberg, J.T.: The Essential Physics of Medical Imaging. Lippincott Williams \& Wilkins (2002) ISBN 0683301187

2. Mailloux, G., Bertrand, M., Stampfler, R., Ethier, S.: Local histogram information content of ultrasound B-mode echographic texture. Ultrasound in Medicine and Biology, Vol. 11, (1985) 743-750.

3. Mailloux, G., Bertrand, M., Stampfler, R., Ethier, S.: Computer Analysis of Echographic Textures in Hashimoto Disease of the Thyroid. Journal of Clinical Ultrasound, Vol. 14, (1986) 521-527.

4. Chikui, T., Okamura, K., Tokumori, K., Nakamura, S., Shimizu, M., Koga, M., Yoshiura, K.: Quantitative analyses of sonographic images of the parotid gland in patients with Sjögren's syndrome. Ultrasound in Medicine and Biology, Vol. 32, (2006) 617-622.

5. Raeth, U., Schlaps, D., Limberg, B., Zuna, I., Lorenz, A., Kaick, G., Lorenz, W., Kommerell, B.: Diagnostic accuracy of computerized B-scan texture analysis and conventional ultrasonography in diffuse parenchymal and malignant liver disease. Journal of Clinical Ultrasound, Vol. 13, (1985) 87-99.

6. Llobet, R., Pérez-Cortés, J., Toselli, A., Juan, A.: Computer-aided detection of prostate cancer. International Journal of Medical Informatics, Vol. 76, (2006) 547-556.

7. Vince, D.G., Dixon, K.J., Cothren, R.M., Cornhill, J.F.: Comparison of texture analysis methods for the characterization of coronary plaques in intravascular ultrasound images. Computerized Medical Imaging and Graphics, Vol. 24, (2000) 221-229.

8. Ojala, T., Pietikäinen, M., Harwood, D.: A comparative study of texture measures with classification based on featured distribution. Pattern Recognition, Vol. 29, (1996) 51-59.

9. Keramidas, E.G., Iakovidis, D., Maroulis, D., Karkanis, S.A., Efficient and Effective Ultrasound Image Analysis Scheme for Thyroid Nodule Detection. Lecture Notes in Computer Science, Vol. 4633, (2007) 1052-1060. 
10.Caballero, K., Barajas, J., Pujol, O., Savatella, N., Radeva, P.: In-vivo IVUS Tissue Classification A Comparison Between Normalized Image Reconstruction and RF Signals Analysis Progress in Pattern Recognition. Image Analysis and Applications, Vol. 4225, (2006) 137-146.

11.Rotger, D., Radeva, P., Rodriguez, O., Mauri, J.: Near Real-Time Plaque Segmentation of IVUS. Computers in Cardiology, Vol. 30, (2003) 69-72.

12.Brunenberg, E., Pujol, O., Romeny, B.H., Radeva, P.: Automatic IVUS segmentation of atherosclerotic plaque with Stop \& Go snake. Medical Image Computing and ComputerAssisted Intervention, Vol. 4191, (2006) 9-16.

13. Vapnik, V.: Statistical Learning Theory. New York: Wiley. (1998) ISBN: 978-0471030034.

14.Theodoridis, S., Koutroumbas, K.: Pattern Recognition. Academic Press, (3nd edition). (2006) ISBN: 0-12-369531-7

15.Skouroliakou, C., Lyra, M., Antoniou, A., Vlahos, L.: Quantitative image analysis in sonograms of the thyroid gland. Nuclear Instruments and Methods in Physics Research, Vol. $569,(2006) 606-609$. 